\title{
Emission inventory for an urban area: construction and use
}

\author{
E. Brizio, G. Genon \& S. Caon \\ Turin Polytechnic, Italy
}

\begin{abstract}
The situation of air pollution due to nitrogen oxides in an urban area of North Italy has been evaluated starting from the definition of emission scenario calculated on the basis of specific localisation of emitting sources, their working regime and emission factors. Afterwards, by using specific atmospheric models suitable to the different sources and localisations, it was possible to evaluate the concentration maps; it was also necessary to consider the presence of a stagnating quantity of pollutants forming a background concentration that has been evaluated by means of experimental data. It was possible to ascertain that the traffic contribution, which by itself is quite important from the point of view of the emissive scenario, is the dominant factor as far as the reduction in air quality is concerned. On the basis of this observation, some traffic limitation interventions have been considered, and it was interesting to establish their contribution on air quality. The methodological path that has been constructed presents a numerical significance that is completely site-specific, but it can also be considered a useful tool for the political authority; in fact, on the basis of the correct knowledge of an emissive scenario and a valid atmospheric model, it is possible to set up proper policies for an air quality improvement.

Keywords: traffic, nitrogen oxides, atmospheric model, urban area.
\end{abstract}

\section{Introduction}

The air pollution situation of many European urban areas does not present indications of any substantial improvements, in spite of the adoption of technological interventions for emission limitations and processes for source reduction (CAFE Programme, 2005); actually, these actions, without other activities, such as a clear understanding of emissive and atmospheric phenomena 
influencing the result, are not able to lead the air quality back to desired standards.

In order to deal with the problem in the right way, many subsequent elements are necessary, as follows:

- it is necessary to individuate the principal emission fluxes, taking into account the sources spatial distribution and their capacity to generate fixed quantities of pollutants;

- the correlation between emitted fluxes and environmental concentrations must be evaluated by means of atmospheric models, and the results must be compared with experimental values;

- with reference to different emission scenarios, the different effect on air quality must be established, and the obtained concentrations must be evaluated and compared to the required standards; this way, it will be possible to establish criteria for real time limitation or structural interventions.

The above described approach requires, for the first two steps, to overcome some obstacles; with reference to the first task, it is necessary to construct a realistic inventory of emissions produced from different activities, and this inventory must be disaggregated with reference to times and local situations; chiefly for some pollutants (like nitrogen oxides, that will be the subject of this article, while different should be the evaluations about fine dusts) there are not conceptual difficulties for this evaluation, but it is important to underline and stress the attention that must be devoted to collection and organisation of existing technological and territorial data.

In order to study the impact aspects by means of atmospheric models, the influencing mechanisms are completely known and conceptually defined; nevertheless it is necessary to calibrate the models with reference to specific territorial situation, by considering meteorological aspects, spatial and topographic collocation of sources and objects that can hinder the free circulation of air masses; chiefly in urban areas the last one seems to be the most important argument of investigation that must be dealt with. Finally the third step substantially corresponds to an activity of political decision on the basis of verified information and reliable predictions deriving from the individuated scenario; for this step the fundamental role belongs to public authorities, but it must be developed on the ground of solid technical information.

The so defined route will be presented with reference to a city in the North of Italy, where it is strictly necessary to adopt limitation policies; the presence of emissions, meteorological and air quality data is the precondition in order to perform a process of definition and calibration of models, and subsequently to arrive to the definition of improvement scenarios.

The meaning of the described process is highly methodological, as in any territorial situation emission inventories and atmospheric dispersion capacities are different and strongly dependent on the specific area; nevertheless this approach, constructed and calibrated for a specific case, can be usefully extrapolated also to other urban situations. 


\section{Investigated area, emission and air quality data}

The investigated area corresponds to the town of Alba, a middle dimension city (40.000 inhabitants) of North of Italy, where the presence of pollutants (chiefly nitrogen oxides, dusts, ozone) is quite high as a consequence of heavy road traffic, large industrial activities, and domestic heating. The most convenient parameter adopted for the study has been identified in nitrogen oxides, because it represents a critical point in the investigated area, and at the same time one has the possibility of individuating the technological origin of this pollutant. As far as the data that have been used as a basis for the study are concerned, they are as follows.

Meteorological data. From the local Environment Protection Agency it was possible to obtain for a whole solar year (the year 2004 in particular) for two urban stations data about wind direction and wind speed, temperature, solar radiation; these data have been organised with statistic approach as far as the convective component is concerned, while the stability classes have been defined using the SRDT scheme obtained from U.S. EPA; as for the mixing height, with a similar approach, we used screening values provided by U.S. EPA for night or day conditions and different stability classes.

Topographic data. In the main urban streets the traffic fluxes were known, and there the buildings geometry has been evaluated; this way, on the basis of the structure of surrounding buildings, in some cases the geometry has been defined as an urban canyon; otherwise, where the buildings are low, not close to the street or not present, an open spatial structure was considered.

Air quality data. For the year 2004 we have at disposal the measured hourly data of nitrogen oxides concentration in an urban point. The localisation corresponds to a backyard, and so it is possible to consider this series of registrations as representing mean values for the city; as a matter of fact, there is very low influence of local effects, and on the contrary it is possible to identify there an overlapping of the effects of different emitting sources.

Emission data. First of all the contribution deriving from road traffic has been evaluated; the Alba municipality provided hourly values of circulation of different vehicle types for the main streets, and from these values, taking into account the emission factors derived from COPERT III program (Ntziachristos and Samaras [2]) and the length of considered street stretches, it was possible to define linear emissions; when using COPERT III we obviously considered the composition of the vehicle park in different emissive classes, as required from the program. As for the domestic heating, the starting point was the definition of inner volumes where different fuels (natural gas, gas oil, fuel oil) are used, peak load, daily energy distribution and annual volumetric thermal requirement, as indicated by local energy data (Sordo and Calì [3]), and emission factors indicated in EPA database were applied; the emissions corresponding to heating were subsequently distributed on a limited number of discrete sources, whose dimensions were defined from inhabitants distribution. Afterwards it was necessary to consider the presence in Alba territory of a district heating station, 
formed by four boilers and five internal combustion engines; for these apparatus the power, emission capacity, energetic production in different seasons were known. Finally, the emission of a large food production factory operating in the urban territory was considered; also in this case the emissive and operating features of the three large boilers of the plant were known. The last two emissions (district heating station and industrial plant) in the emissive scenario have been considered point sources.

On the basis of the above-mentioned considerations it was possible to define the hourly values of different nitrogen oxides emissions, and the total yearly values; in Figure 1, on the left, the percent distribution of different NOx emission sources is shown, while, on the right, as far as the traffic is concerned, the contribution of different vehicle types is reported. From these figures it is possible to verify that the traffic input, and chiefly cars, is absolutely the predominant one, as far as emitted fluxes are concerned; in the following point the effect of different sources on air quality will be discussed.

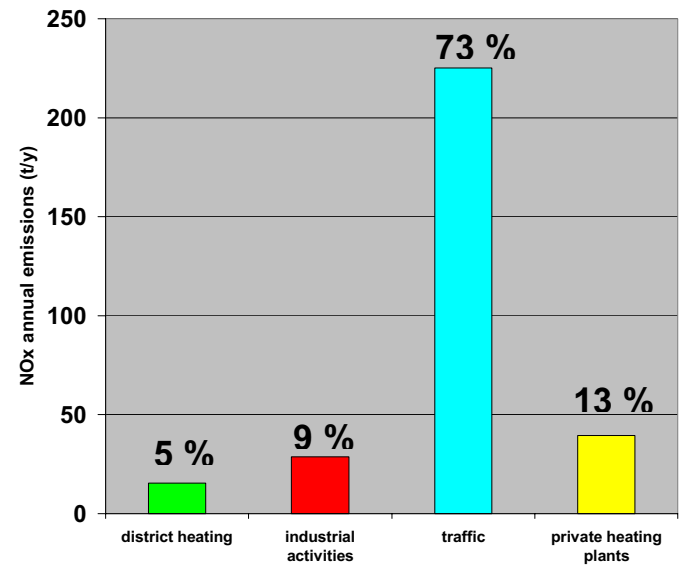

\section{TRAFFIC}

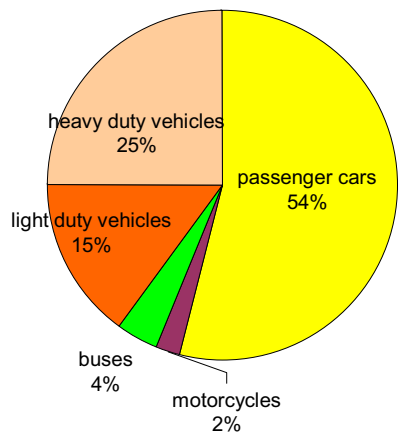

Figure 1: NOx emission sources in Alba.

\section{Model calculation of environmental concentrations}

In order to define environmental concentrations of NOx in different meaningful city points, it was firstly considered the possibility to use existing atmospheric dispersion models, taking into account the urban configuration where the models must be applied, and the localisation of emission points considered as sources.

As for the emissions deriving from linear traffic sources, situations where an urban canyon structure should be assumed were distinguished from more open roads conditions; in the first case the OSPM model (Berkowicz et al. [4]) has been used, as this model describes the concentration deriving from exhaust gases using a combination of a plume model for the direct contribution and a box model for the recirculating part of the pollutants in the street, because of the 
vortex structure usually formed in the canyon. On the contrary for open roads the more suitable model was ISCST3 (US EPA [5]), in account of its capacity as a conventional steady-state plume Gaussian model to describe a transport and turbulent dispersion condition.

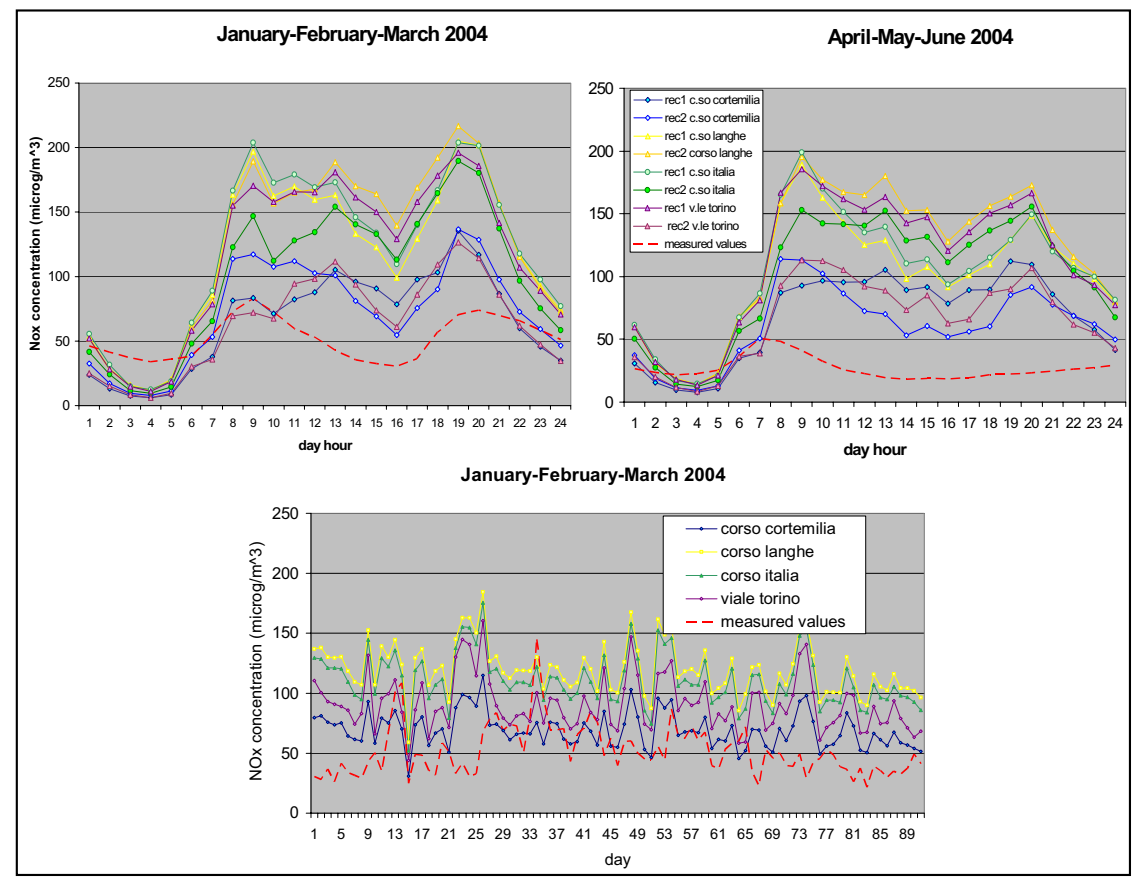

Figure 2: $\quad$ NOx calculated concentrations for street canyons in Alba.

In Figure 2 the results obtained by means of the OSPM model in different day hours and for different season periods are reported (for each considered street two values are reported, i.e. the leeward and the windward side of the street); in the lower part of Figure 2 the daily average concentrations are represented; in the plot the red dashed line represents the measured concentrations at the station located in a particular area of the city where the influence of local traffic is low.

As for the hourly concentration values, it is possible to establish that the calculated trends are very strictly influenced by the traffic fluxes, as maximum values are observed when the traffic peak is realized; on the contrary during the night the calculated levels are very low, when higher values could be observed for the measurements station, as a consequence of a stagnation phenomenon that cannot be represented by the steady-state type model defining the correlation between emission and concentration. The mean daily values show a similar trend, with very high differences chiefly in summer period between absolute calculated values for busy streets and measured values of the station (placed, as previously said, in a quiet zone); during the months between April and 
September, the qualitative trends are similar, including relative minimum and maximum values, and so it can be confirmed the important effect of traffic contribution; during the winter, on the contrary, when the heating contribution effect cannot be disregarded, it is possible that the measured values exceed the model calculated ones.

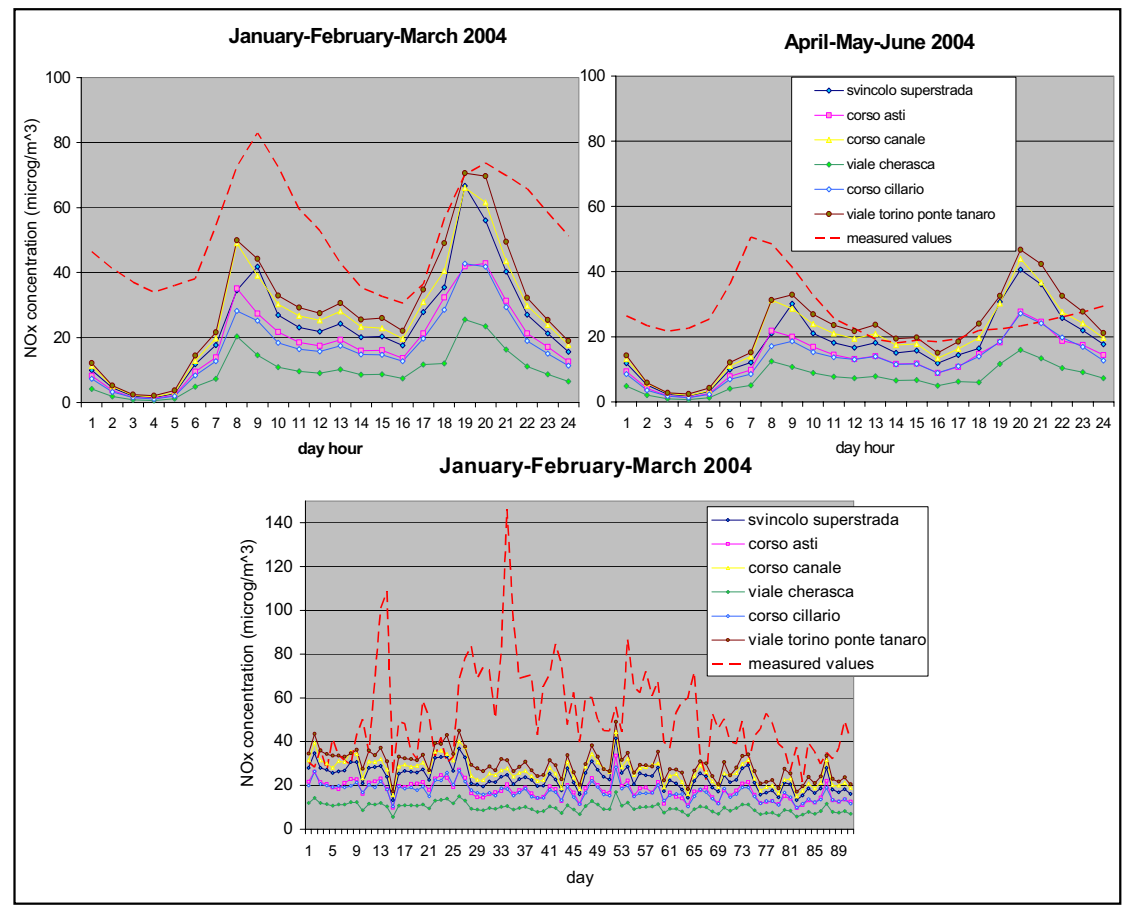

Figure 3: NOx calculated concentrations for non canyon streets in Alba.

Figure 3 reports similar results for the non canyon type, more open streets, where the ISCST3 model was used; it is possible to remark that the trend of values calculated during the day leads to lower concentrations, in account of the higher dispersion capacity of the considered zones; in the same way, as far as the daily mean values are concerned, the plots of calculated concentrations are qualitatively different from the measured ones, mainly during the winter time, and this fact is a confirmation of the different influence of source factors on the trends.

The dispersion of pollutants arising from domestic heating plants, the district heating boilers and engines and the industrial plants can be described by using ISCST3 model, taking into account the thermal plume rise effect; Figure 4 reports the seasonal mean concentrations for the domestic heating plants, whereas the concentration maps for the district heating and the industrial emissions refer to the yearly average. 


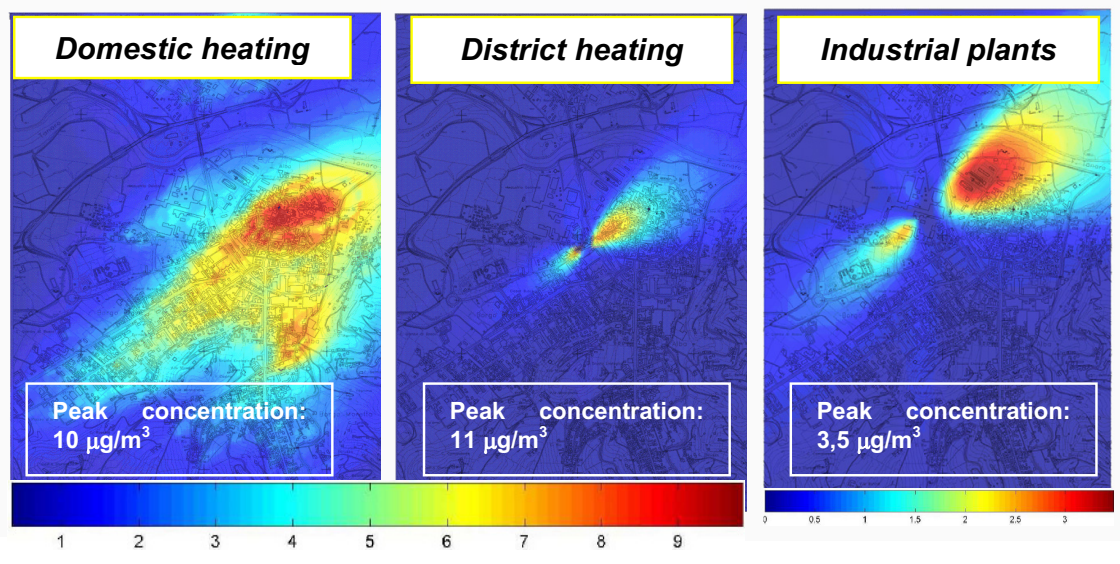

Figure 4: $\quad$ NOx concentration map for the other sources.

The analysis performed by using these models takes into account the effect of the actual emission on actual concentrations, but the stagnation effects are disregarded; it should be considered that the emitted substances remain for some times in the atmosphere, and they are added to the concentration that is immediately created by emitting sources. In order to individuate the background concentration, as a first approximation, we considered the concentrations measured at the station between $24 \mathrm{pm}$ and $5 \mathrm{am}$, with virtually absent traffic, and the contributions from district heating system and industrial plant were subtracted. In the winter, the mean value of the difference for the 5 hours has been identified as a daily background mean value; during the summer, in order to take into account the effects of higher dispersion (larger mixing height) and photolytic transformations, it was assumed that the background contribution had a lower effect on the mean daily concentration; so the determined background concentration has been corrected by the numerical factor $5 / 24$ during the period from May to September.

Figure 5 reports the comparison between the simulated concentrations (due to traffic, district and domestic heating, industrial sources) summed with the background concentrations calculated in the described way and the measured values for a non canyon street. As one can easily observe, in this case the results (expressed as 15 days running average) are very satisfactory; on the contrary, for a canyon street the behaviour of the calculated concentrations follows quite well the measured ones but an important shift is evident.

By taking into account the different so-defined contributions, it was possible to calculate the fraction of the total concentration due to each of them. These estimations are reported in Figure 6, in the left side for a typical canyon street, and in the right side for an open street; it is possible to observe that the traffic contribution to the determined concentration has an influence of $80 \%$ in the first configuration, and of 50\% in the second one. Anyway it is necessary to take into account that the background contribution, if it is distributed in the same way as the original sources, leads to an estimation of a very low effect of the substances 
deriving from fixed sources on the air quality in the examined case, and this effect is also lower if compared with their contribution in emissive terms.

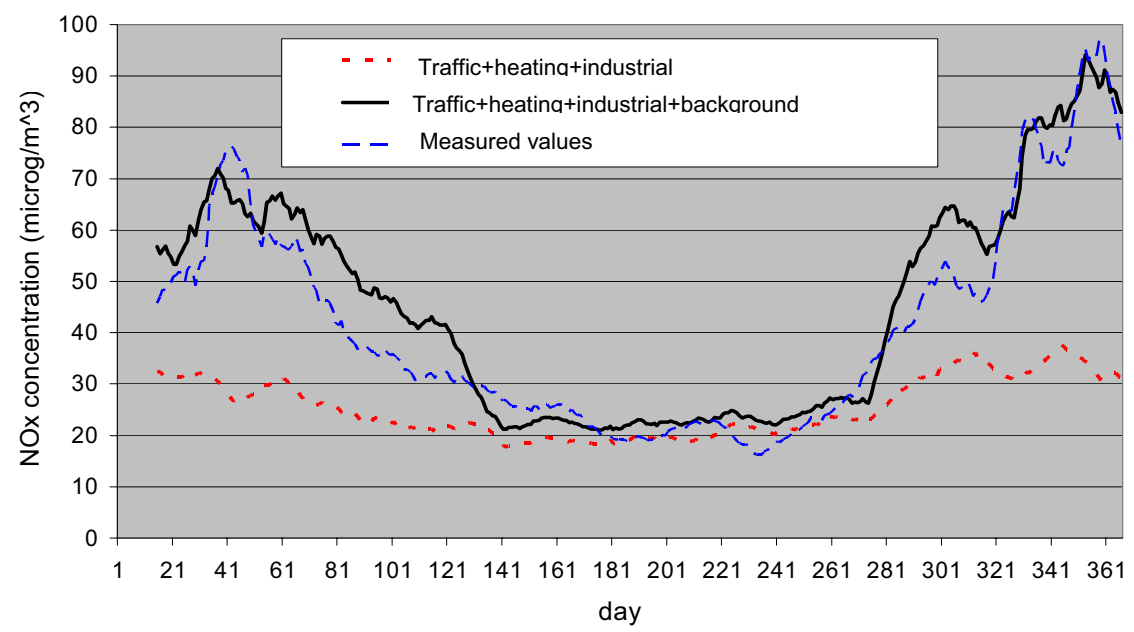

Figure 5: Comparison between calculated and measured concentrations in a non canyon street.

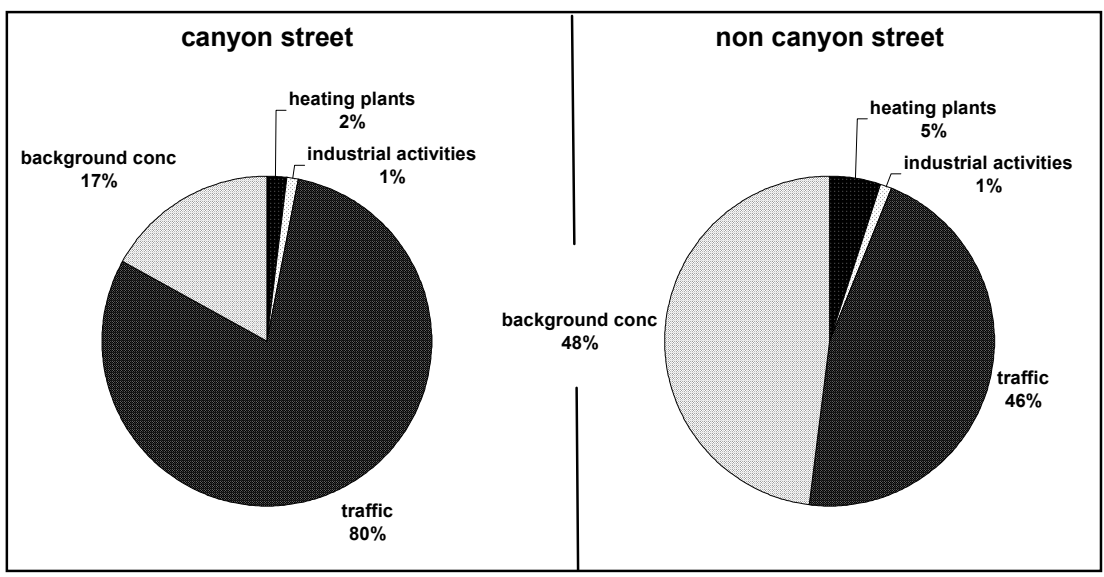

Figure 6: Contributions of different sources to the calculated concentrations.

\section{Strategies for pollution reduction}

The described model approach has been then used in order to put into evidence the effects of traffic limitation policies (that is the most important pollution 
factor) on the air quality levels. In particular we considered the possibility, with these limitation interventions, of reducing the concentration levels below the allowed air quality limits. In order to correctly evaluate this consideration, it is in any case important to underline that the comparison can represent only a suggestion, and not a final statement, as the emission and dispersion model considers only the formed NOx, essentially in NO form, while for the air quality standards the considered parameter is $\mathrm{NO}_{2}$; in order to obtain a more realistic comparison, we should introduce a photochemical transformation parameter, and validate this parameter on the local scale, and this aspect is not usually at disposal today; in any case it is possible to present the comparison at least as an indication and as a method illustration.

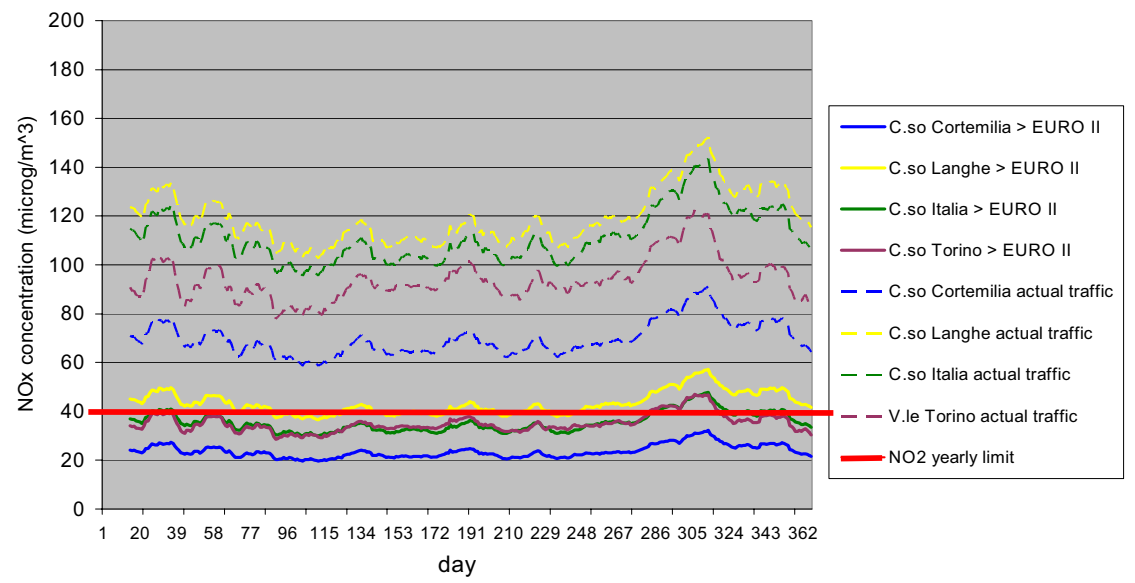

Figure 7: Effects of a traffic limitation policy on the air quality.

Figure 7 shows the results of a particular simulation, the phasing-out of the vehicles belonging to a class before EURO II; in the plot, the dashed lines report the value corresponding to unmodified car park; as it can be observed, the simulated reduction strategy allows to obtain mean year values lower than the regulation limits $\left(40 \mu \mathrm{g} / \mathrm{m}^{3}\right)$, with some very limited exceptions for a particular high traffic road.

The described use of dispersion models, with the possibility of modifying the pressure factors, represents an important tool for the prediction of the effects of scenario modifications on air quality; a similar application, that cannot be reported here for space reasons, was also obtained by studying the expansion of district heating and the realisation of further cogeneration systems, located within the industrial production plant present in the town; also in this case it was interesting and meaningful to predict, on the basis of some modifications of emissive scenarios, the variation of calculated ambient concentrations, and chiefly the effects on determined maximum values. 


\section{Conclusions}

The results of this study were able to very clearly demonstrate the critical aspects for the area taken into account, but at the same time they can be considered a methodological approach in order to evaluate pressure factors, their transfer effect, the possibilities of intervention on the origins. The strength of the traffic effect can be considered the most meaningful obtained information, and it is an important confirmation of the authorities intervention directives; it is in particular useful to extract a reasoned decision support from the results.

Within a more general point of view, as an indication of the methodologies to be used, some points requiring a deeper definition have been identified.

The emission inventory. While some parameters and some technological sources require only a careful and detailed data collection effort, for other parameters (as for example fine dusts or volatile organic substances), both the diffuse emission, (due to technological but also natural origins), and the formation mechanisms need further investigation, also by introducing experimental determinations;

Atmospheric modelling of an urban area. This aspect is very complicated in account of complex fluid dynamic regime and the particular geometry of the physical system; in account of these aspects specific studies supported by experimental validation are required in order to consolidate the knowledge of spatial and time correlation between emission scenario and concentration fields. Moreover for some not conservative parameters (the photochemical smog phenomena are an example of this aspect) the physical dispersion and convection model by itself doesn't seem enough to evaluate the pertinent phenomena.

On the basis of the above-indicated conclusions it is very important to continue the outlined studies, as they seem to be the unique valid instrument for a correct definition of intervention strategies for urban pollution problems.

\section{References}

[1] Amann, M. Bertok, I. et al, Baseline Scenarios for the Clean Air for Europe (CAFÉ) Programme, February 2005.

[2] Ntziachristos, L., Samaras, Z., COPERT III Computer programme to calculate emissions from road transport, European Environmental Agency, 2000.

[3] Sordo, S., Calì, M., Analisi energetica ed ambientale del progetto di teleriscaldamento di Mondovi, Politecnico di Torino, 2005.

[4] Berkowicz, R., Olesen, H., Jensen, S., Operational Street Pollution Model, User's Guide to Win OSPM, National Environmental Research Institute, Denmark, 2003.

[5] U.S. EPA, User's guide for the Industrial Complex (ISC3) Dispersion Models, Research Triangle Park, North Carolina 27711, 1995. 